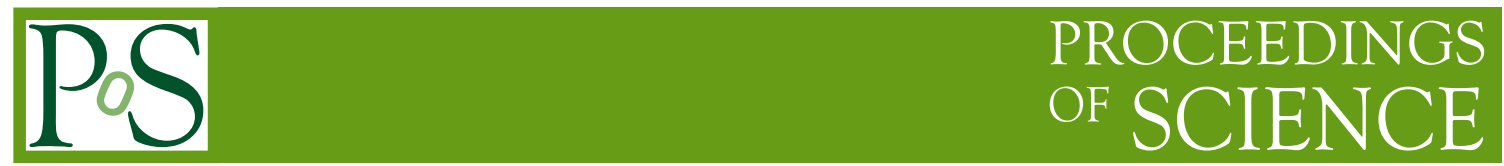

\title{
Performance of the reconstruction and identification of high-momentum muons collected with CMS in $13 \mathrm{TeV}$ data
}

\section{Carlo Battilana*, on behalf of the CMS Collaboration}

Università di Bologna ed Istituto Nazionale di Fisica Nucleare (INFN) - Sezione di Bologna, viale Berti Pichat 6/2 - 40127 - Bologna, Italy.

E-mail: carlo.battilana@cern.ch

The CMS detector at the LHC has recorded events from proton-proton collisions, with muon momenta reaching up to $1.8 \mathrm{TeV}$ in the collected dimuon samples. These high-momentum muons allow direct access to new regimes in physics beyond the standard model. Because the physics and reconstruction of these muons are different from those of their lower-momentum counterparts, this talk presents the first dedicated studies of efficiencies, momentum assignment, resolution, scale, and showering of very high momentum muons produced at the LHC.

40th International Conference on High Energy physics - ICHEP2020

July 28 - August 6, 2020

Prague, Czech Republic (virtual meeting)

${ }^{*}$ Speaker 


\section{Introduction}

During its second run period (Run-2), lasting from 2015 to 2018, the Large Hadron Collider (LHC) was able to deliver proton-proton collisions at a centre of mass energy $(\sqrt{s})$ of $13 \mathrm{TeV}$, and reached instantaneous luminosities up to $\sim 2.2 \cdot 10^{34} \mathrm{~cm}^{-2} \mathrm{~s}^{-1}$. Over Run-2, the Compact Muon Solenoid (CMS) recorded around $160 \mathrm{fb}^{-1}$ of such high energy collisions, $140 \mathrm{fb}^{-1}$ of them have been validated for physics analyses. The increase of $\sqrt{s}$, as well as the larger amount of data collected with respect to the LHC Run-1, allowed to extend significantly the reach of searches for new physics involving high-invariant-mass Beyond-the-Standard-Model (BSM) particle candidates. The signatures of several of these analyses are characterized by final states involving muons with energies of few hundreds $\mathrm{GeV}$ or more. The large number of high-energy muons collected over Run-2 allowed to study, with unprecedented accuracy, the performance of the algorithms used to reconstruct, identify, and trigger on them.

\section{Muon reconstruction and identification in CMS}

The CMS muon spectrometer and the muon reconstruction algorithms are documented in [1]. Muon tracking relies on the detection of a particle's trajectory along the multiple layers of the silicon inner tracker, immersed in a $3.8 \mathrm{~T}$ solenoidal magnetic field, and within the four concentric stations rings of gaseous detectors which build the muon system, hosted in the inside the steel return yoke of the CMS magnet. The inner tracker, covering a pseudorapidity $(\eta)$ range $|\eta|<2.5$, consists, in its inner and outer parts, of pixel and strip detectors respectively. The muon system presently covers $|\eta|<2.4$ and, over Run-2, it consisted of three different detector technologies. Drift Tubes (DT) equip the spectrometer barrel $(|\eta|<1.2)$ whereas Cathode Strip Chambers (CSC) are installed in the two endcaps $(0.9<|\eta|<2.4)$ of the muon system. Both are tracking devices with self-trigger capabilities and consist of chambers built of multiple detector layers. They are complemented by Resistive Plate Chambers (RPC), ensuring robustness to the muon trigger up to $|\eta|<1.9$.

The reconstruction of muons starts by forming hit clusters in the pixels, strips and RPCs, whereas, in each DT and CSC chamber, the reconstruction of straight-line segments is attempted. Out of these basic components, tracks are built in parallel in the inner tracker (inner tracks) and in the muon system (standalone tracks). For each standalone track, a set of nearby tracker tracks is identified and, after propagating all of them to a common surface, a matching is attempted by testing the compatibility between the former and each of the latter ones. In case of success, the best pairing is retained and a combined fit of all available hits and segments is performed producing a global-muon track. Furthermore, an inside-out muon identification algorithms is also attempted. Inner tracks which are above a minimum energy (and transverse momentum) threshold, are propagated to the different stations of the muon system, with an algorithm that accounts for energy loss and multiple scattering effects. At each crossed station, every track gets probed for geometrical matching with CSC and DT segments. A tracker muon is built each time one or more segments are found to be compatible with an extrapolated tracker track.

Reconstructed muons also provide a set of variables to define, so called, identification and isolation criteria, which allow each analysis to tune the balance between efficiency and purity and optimise the selection of muons according to the probed phase-space. These variables include: 
(i) quality parameters from the muon reconstruction itself (e.g. the number of inner tracker layers used to form the muon track, the number of stations with DT/CSC segment matches in a tracker muon, ...); (ii) the compatibility of a muon track with the primary collision vertex; (iii) the energy sum of tracks, calorimeter deposits or particle-flow candidates in cones surrounding the muons.

\subsection{Reconstruction and fitting of high-energy muons}

For muon energies above a few hundreds $\mathrm{GeV}$, the curvature of the particle trajectory gets reduced and the resolution of the transverse momentum $\left(p_{\mathrm{T}}\right)$, measured at lower momenta using solely the inner track component of a reconstructed muon, degrades. At such scales, multiple scattering gets also smaller, therefore combining information from the inner tracker and the muon system within a track fit significantly improves the $p_{\mathrm{T}}$ resolution, as shown in Fig. 1 (left).

Of course, in case of small track curvature, inaccuracies in the measurement of the position (and direction) of hit clusters (segments), due to a non perfect alignment of the inner tracker (muon system) can significantly bias the measurement of the muon $p_{\mathrm{T}}$. It is hence essential to accurately align the detector (i.e. the position of a DT/CSC segment in the radial coordinate must be known with a precision of $100-200 \mu \mathrm{m})$.
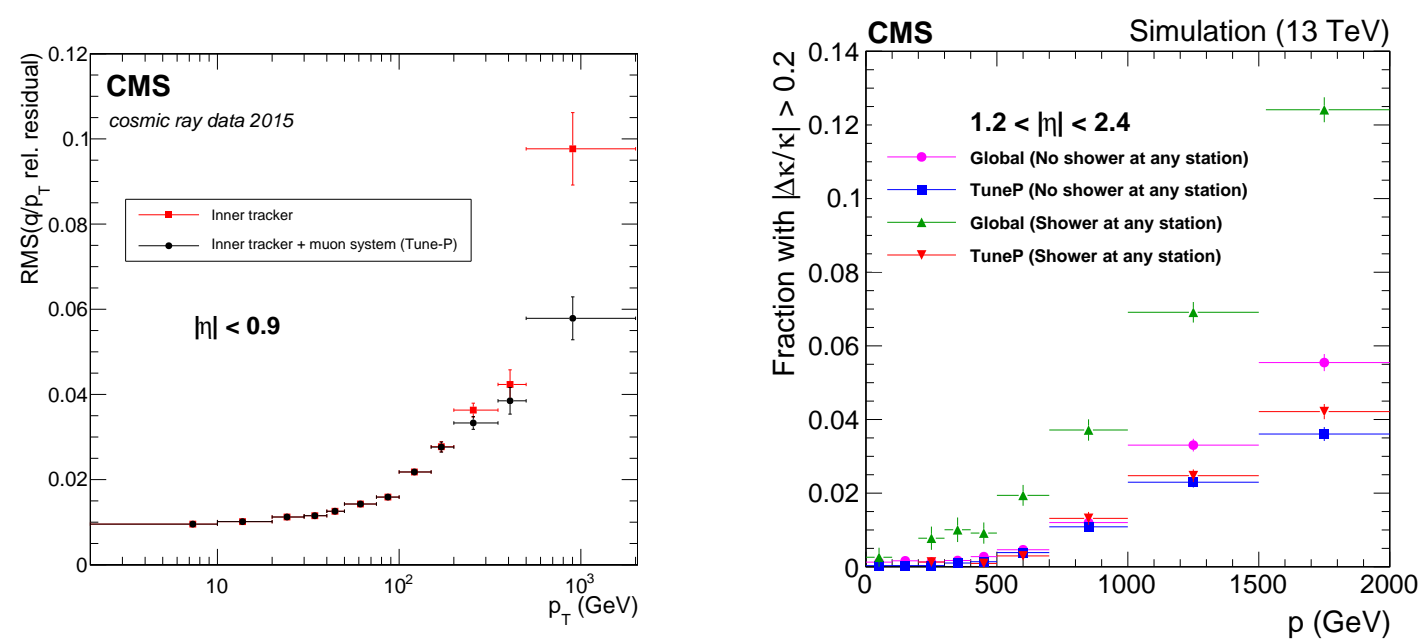

Figure 1: Muon transverse momentum assignment resolution performance. Left: comparison between the resolution of muon track fits exploiting solely hits from the inner tracker (red) and combining information from the inner tracker and the muon system (black). Right: fraction of events where the track curvature is measured with an error larger than $20 \%$ in case the global fit or the tune-P refit are used, also considering events where showers are identified in the muon system.

Furthermore, for high momentum muons traversing the steel yoke of the CMS magnet, radiative energy losses become sizable with respect to losses from ionization. In the case of iron, the two are equivalent around $300 \mathrm{GeV}$, with the former dominating over the latter at higher energies. Therefore, methods to identify when such events occur have been studied, and strategies to account for showering effects at the muon track fitting stage have been developed. In the following, we exploit a shower-tagging logic that, in each muon system station, counts eventual extra DT or CSC segments which are present, on top of the one participating to the muon track, in the vicinity of the muon trajectory. Though this empirical method is not perfect (i.e. it does not capture showers 
which are entirely contained in the steel magnet yoke, and it is based on arbitrary threshold cuts), it provides a powerful tool to categorize cases where large multiplicity of hits occur in proximity of a muon track and study them.

Moreover, in presence of electromagnetic showers, the $p_{\mathrm{T}}$ estimated from the global muon fit (which is designed to retain very high hit association efficiency), might occasionally turn out to be significantly inaccurate, resulting for example in tails in the measurement of the $p_{\mathrm{T}}$ resolution. For this reason several algorithms, targeting an optimal combination of muon system segments and inner tracker hits in the muon track fit, and aiming at improving the muon $p_{\mathrm{T}}$ resolution in specific cases, were developed. The simplest strategy, called track-plus-first-muon-station (TPFMS) fit, combines the information from the inner track with the one of the innermost muon station where the global muon fit has identified a compatible segment. This extends the lever-arm of the fit, while minimizing the possibility of contamination from showers. Instead, the picky fit retains information from all segments from the global muon track, unless for cases where a large multiplicity of hits is found in a given muon system chamber. If that occurs, more stringent requirements of hit compatibility to the muon track are applied, and information from a given chamber is rejected if they are not met. Furthermore, the dynamic truncation (DYT) fit starts from the inner track and propagates it outwards, adding iteratively DT and CSC segments which are found compatible with the track trajectory. If no compatible segments are found in two consecutive stations (e.g. in case of a severe kink due to a shower) the iterative process stops. Finally, based on the quality metrics from either the inner-track fit or the track-fit strategies described above (such as the $\chi^{2}$ or the relative $p_{\mathrm{T}}$ error), the tune- $P$ algorithm chooses which of them is going to be used to determine the muon transverse-momentum. In cases where the final candidate has a $p_{\mathrm{T}}$ of $200 \mathrm{GeV}$ or less, the innertrack fit is preferred to other refits. Figure 1 (right) proves the effectiveness of the strategy, showing that the tune-P $p_{\mathrm{T}}$ assignment reduces significantly the fraction of events with a $p_{\mathrm{T}}$ mis-assignment larger than $20 \%$, if compared to the global fit. Results are provided for the muon system endcap, and are especially remarkable in the case of shower-tagged events.

\section{Performance}

The performance to reconstruct and identify high-energy muons, masured using data collected by CMS during the 2016 and 2017 LHC proton-proton collision runs, is documented in [2]. Furthermore, the analyses that depend more significantly on the update on the detector alignment were repeated, to validate reprocessings performed with updated calibration conditions [3].

\subsection{Reconstruction, identification and trigger efficiency}

The efficiency to reconstruct, identify and trigger on high-energy muons is measured in data and simulations by means of an extended tag-and-probe technique. As for standard tag-and-probe analyses [1], the method exploits dimuon decays from DY events which are triggered by a tag muon, that is also requested to satisfy stringent identification and isolation criteria. Though, oppositely to the tag-and-probe strategy used at lower energies, the contribution of probe muons from the highmass non resonant tail of the DY spectrum is enhanced by requiring a balance between the $p_{\mathrm{T}}$ of tags and probes. Furthermore, in all studies, the probe is requested to satisfy tight isolation cuts. In this way the purity of the sample was tested to be high enough to allow for a cut and count efficiency 
measurement. The definition of denominator (probes) and numerator (passing probes) used to measure the efficiency varies depending on the component of the muon selection to be studied. The reconstruction efficiency is $99 \%$ or above, flat as function of momentum, up to $|\eta|<1$.6. Instead, a momentum dependent inefficiency, up to $3 \%$, is measured at larger $|\eta|$. The identification efficiency is found to be mostly above $98 \%$, with no significant energy dependence across the full detector, as shown for $1.2<|\eta|<2.1$ in Fig. 2 (left). Finally, the trigger efficiency varies approximately from $92 \%$ to $82 \%$ in the momentum range from $50 \mathrm{GeV}$ to $1 \mathrm{TeV}$, with data/simulation agreement ranging between $95 \%$ and $90 \%$, as shown, for $|\eta|<0.9$, in Fig. 2 (right).
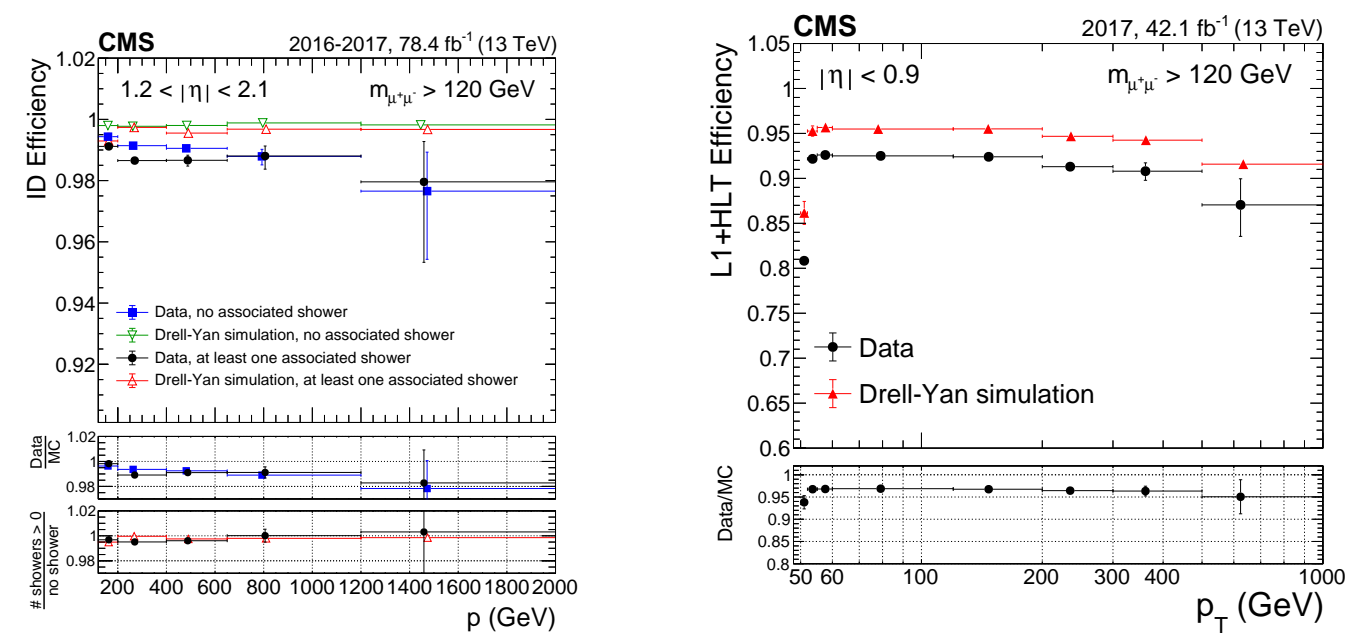

Figure 2: Muon identification and trigger efficiency measured with the extended tag-and-probe method. Left: muon identification efficiency in the endcap region of the muon spectrometer measured in data and simulation both considering cases where no showers, or at least one shower were identified in the muon system. Right: overall trigger efficiency measured in the barrel region of the muon spectrometer for data (black) and simulation (red).

\subsection{Muon momentum scale and resolution}

The resolution of high energy muons is computed both by exploiting boosted dimuon decays from $Z$ bosons, and cosmic ray data. The latter is characterised by a harder $p_{\mathrm{T}}$ spectrum compared to the former, but covers effectively only the muon system barrel. In the first case, the width of the $\mathrm{Z}$ resonance is measured separately for different $\eta$ and $p_{\mathrm{T}}$ bins of the highest $p_{\mathrm{T}}$ muon of the pair, and it is used as metric to evaluate resolution. Data and simulation are found in good agreement, modulo a $15 \%$ discrepancy at $|\eta|>1.6$ observed only in the 2017 dataset. In the case of cosmic rays, muons traversing the detector from top to bottom in a fiducial region corresponding to the pixel subdetector are reconstructed independently in the top and bottom halves of CMS. The relative $q / p_{T}$ difference between the two muon reconstructed legs is a measure of the $p_{\mathrm{T}}$ resolution. Results from this analysis, presented in Fig. 3 (left), show remarkable agreement between data and simulation.

Biases in the scale of high-momentum muons, arising from an inaccurate measurement of the track curvature, are probed with an analysis called generalized endpoint method. This technique selects dimuon pairs, and the $q / p_{T}$ distribution of muons with a $p_{\mathrm{T}}$ of at least $100 \mathrm{GeV}$ is plotted separately for data and simulation. The distribution falls rapidly for small curvatures, and, if no 
scale biases are present, it has a kinematic endpoint at 0 . In presence of biases due to residual misalignment, typically manifesting as fixed additive terms to the muon curvature, the $q / p_{T}$ distribution gets shifted moving the kinematic endpoint away from 0 . Scale biases are measured in data by injecting, in steps, artificial curvature biases to the $\mathrm{q} / p_{\mathrm{T}}$ distribution obtained from simulation, and comparing the two by means of a $\chi^{2}$ test. The result of the $\chi^{2}$ test is plotted as function of the various scale bias steps injected in simulation and the bias for data is computed by minimizing such distribution. Results, computed in bins of $\eta$ and $\phi$, are presented in Fig. 3 (right), and are compatible with no bias up to $|\eta|<2.1$.
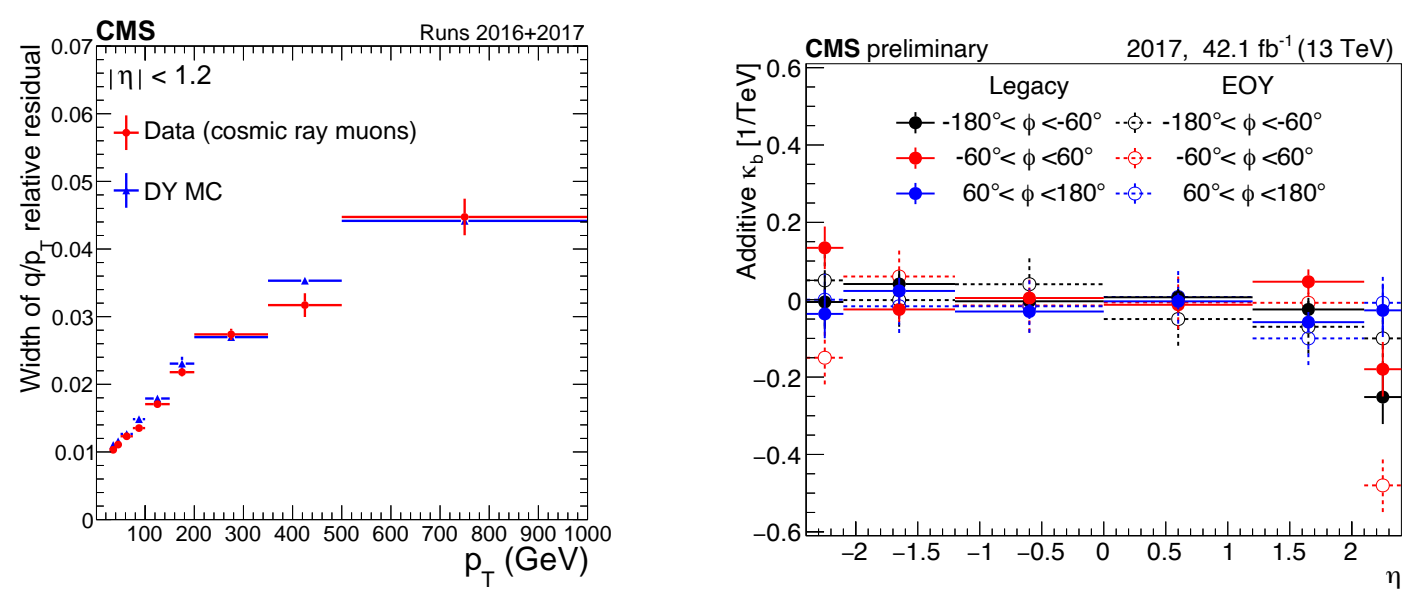

Figure 3: Left: muon transverse momentum resolution, measured using cosmic muons, in data (red) and simulation (blue). Right: muon transverse momentum scale bias, measured by exploiting the generalizedendpoint method. Different colors refer to different bins in radial angle and full and empty markers refer to results from different reprocessings of Run-2 data.

\section{Summary}

Good understanding of high-energy muons is a critical element of many CMS searches for highinvariant-mass BSM particles. Based on data collected over the LHC Run-2, the performance to reconstruct, identify and trigger on such muons was probed, in all of its metrics, to an unprecedented level of precision. Modulo a few minor exceptions, performance in data is found to be remarkable. Furthermore, very good agreement with simulation is observed, also in events where electromagnetic showers are identified.

\section{References}

[1] The CMS Collaboration, "Performance of the CMS muon detector and muon reconstruction with proton-proton collisions at $\sqrt{s}=13 \mathrm{TeV}$ ”, JINST 13 (2018), no.06, P06015.

[2] The CMS Collaboration, "Performance of the reconstruction and identification of highmomentum muons in proton-proton collisions at $\sqrt{s}=13$ TeV”, JINST 15 (2020), P02027.

[3] The CMS Collaboration, "Muon Reconstruction and Identification Performance with Run-2 data”, CMS-DP-2020-040, 2020. 